\title{
Perfiles de expresión génica en decisiones terapéuticas en cáncer de mama
}

\author{
Alejandro Corvalán ${ }^{1,2}$. \\ Gene expression profiles \\ for therapeutic decisions \\ in breast cancer
}

In the early stages of breast cancer, surgery and radiotherapy arrest the local and regional disease. However 30\% of patients will die as a consequence of tumor dissemination. Parameters with a relative predictive value are used to assess candidates to adjuvant therapy. Genomic analysis methods (cDNA microarray) identified a gene expression profile of greater aggressiveness in breast cancer. This knowledge generated the project "Translating Molecular Knowledge Into Early Breast Cancer Management" of the Breast International Group (BIG). This is a consortium of 32 research groups in which Chile participates through the Chilean Cooperative Oncological Research Group (GOCCHI). The project consists in three protocols. In the first, denomimated MINDACT (Microarray for Node Negative Disease may Avoid Chemotherapy), 8000 women will be evaluated by gene expression profile and clinical pathological criteria to decide adjuvant therapy. Since a group of patients will not receive therapy, solely based on gene expression profile, GOCCHI is preparing a follow up protocol based on the expression of 22 genes. MINDACT is the first prospective effort to evaluate gene expression profiles in clinical practice. Our participation in this protocol, installs us in the first line of worlwide oncological investigation (Rev Méd Chile 2007; 135: 257-63).

(Key words: Breast neoplasms; Chemotherapy, adjuvant; Gene expression)

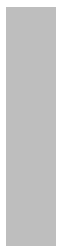

\begin{abstract}
Recibido el 28 de marzo, 2006. Aceptado el 7 de septiembre, 2006.
Financiamiento: Proyectos TRANSBIG-MINDACT, European Union Sixth Framework Programme Proposal, PG 39/04 Pontificia Universidad Católica de Chile y Proy 05/05 Dirección Académica Clínica Las Condes.

${ }^{1}$ Laboratorio Biología Molecular - Departamento Anatomía Patológica, Pontificia Universidad Católica de Chile y ${ }^{2}$ Grupo Oncológico Cooperativo Chileno de Investigación (GOCCHI).
\end{abstract}

$E^{1}$ cáncer de mama es uno de los tumores más frecuentes en el mundo ${ }^{1}$. En la Unión Europea se reportan aproximadamente 135.000 nuevos

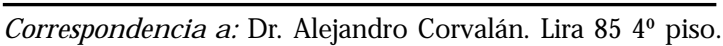
Santiago. casos por año y se registran 58.000 muertes en ese mismo período ${ }^{2}$. En Estados Unidos de Norteamérica, se reportan 215.900 nuevos casos, lo cual representa $30,2 \%$ de todos los tipos de cáncer diagnosticados anualmente y causa 40.110 muertes (16,5\% de todas las muertes por cáncer) por año ${ }^{3}$. En este escenario, Chile no es una excepción, ya 
que el cáncer de mama es la tercera causa de muerte por cáncer en la mujer (mortalidad 12 por 100.000), provocando alrededor de 1.000 muertes anuales ${ }^{4}$.

En los estadios tempranos del cáncer de mama, la cirugía es el tratamiento principal y, en conjunto con la radioterapia, pueden controlar la enfermedad locorregional en la mayor parte de los casos. Sin embargo, alrededor de 30\% de las pacientes finalmente morirá debido a una enfermedad diseminada ${ }^{5-7}$. Es por esto que para erradicar las micrometástasis, prolongar el tiempo libre de recaída y extender la sobrevida global de enfermedad se han desarrollado tratamientos adyuvantes (hormonoterapia o quimioterapia). Para evaluar qué pacientes son candidatas a tratamientos adyuvantes se aplican parámetros clínico-patológicos tales como edad, tamaño tumoral, compromiso de linfonodos axilares, tipo y grado histológico, y receptores hormonales ${ }^{8,9}$. El valor predictivo de cada uno de estos parámetros es relativo, ya que existe una proporción de pacientes en las cuales estas variables no son buenos indicadores de recurrencia ${ }^{10}$. Por ello, actualmente existe una intensa búsqueda de nuevos biomarcadores con mejor poder predictivo de diseminación y tiempo libre de recaída en cáncer de mama.

Los avances en las bases genéticas del cáncer han permitido identificar algunos de los genes responsables de la patogénesis del cáncer de mama $^{11,12}$. Sin embargo, aún no se conocen con exactitud cuáles serían los genes responsables de la progresión del cáncer de mama, los cuales podrían utilizarse como marcadores de diseminación y recurrencia ${ }^{13}$. Entre las estrategias de mayor impacto utilizadas actualmente en la identificación de estos nuevos marcadores son los denominados métodos de alto rendimiento (High- throughput Analysis) ${ }^{14-16}$, que se caracterizan por el análisis masivo de múltiples marcadores genéticos en un solo experimento (entre 100-25.000 análisis en forma simultánea). Algunos de los métodos de High-throughput Analysis, clasificados de acuerdo al tipo de molécula que analizan (ADN, ARN o proteínas) se muestran en la Tabla 1. El más popular de estos métodos es el cDNA microarray que se basa en la hibridación de moléculas de ARN de tejido tumoral contra secuencias del genoma humano impresas en un soporte sólido (láminas de vidrio o membranas de nitrocelulosa) (Figura 1). Para reconocer cuáles son los genes que se están expresando en el tejido tumoral, el ARN del tumor es convertido a cADN y marcado con Texas red, una molécula fluorescente que emite color rojo cuando es excitado con luz en el rango de 650-750 nm. Por otra parte, las secuencias impresas en el soporte sólido están marcadas con FITC, que emite fluorescencia de color verde al ser excitado con luz fluorescente. Una vez producida la hibridación entre el cADN tumoral y las secuencias del genoma humano, los genes sobreexpresados en el tumor se unirán en exceso con respecto a las secuencias del soporte sólido, predominando la señal de Texas red. Por el contrario, si las secuencias del genoma humano no fueron hibridadas por ningún CADN del tumor (probablemente porque el gen no se expresa) predominará la fluorescencia FITC. De este modo, cada lámina 0 membrana permitirá conocer el perfil de expresión génica de un tumor en particular (Figura 1). Esta metodología ha sido aplicada a distintas neoplasias, permitiendo identificar perfiles de expresión génica para la clasificación de leucemias ${ }^{17}$ y cáncer pulmonar ${ }^{18}$ o identificar marcadores de clasificación y pronóstico en cáncer de mama ${ }^{19-22}$. En este sentido, Sorlie

Tabla 1. M étodos de H igh-throughput analysis

\begin{tabular}{|ll|}
\hline Basados en ADN & -Comparative Genomic Hybridization (CGH) \\
Basados en ARN & -Fluorescent in situ hybridization array (FISH array) \\
& -cDNA microarray \\
Basados en proteínas & $\begin{array}{l}\text {-Tissue Microarray (TMA) } \\
\text {-Proteomics }\end{array}$ \\
\hline
\end{tabular}




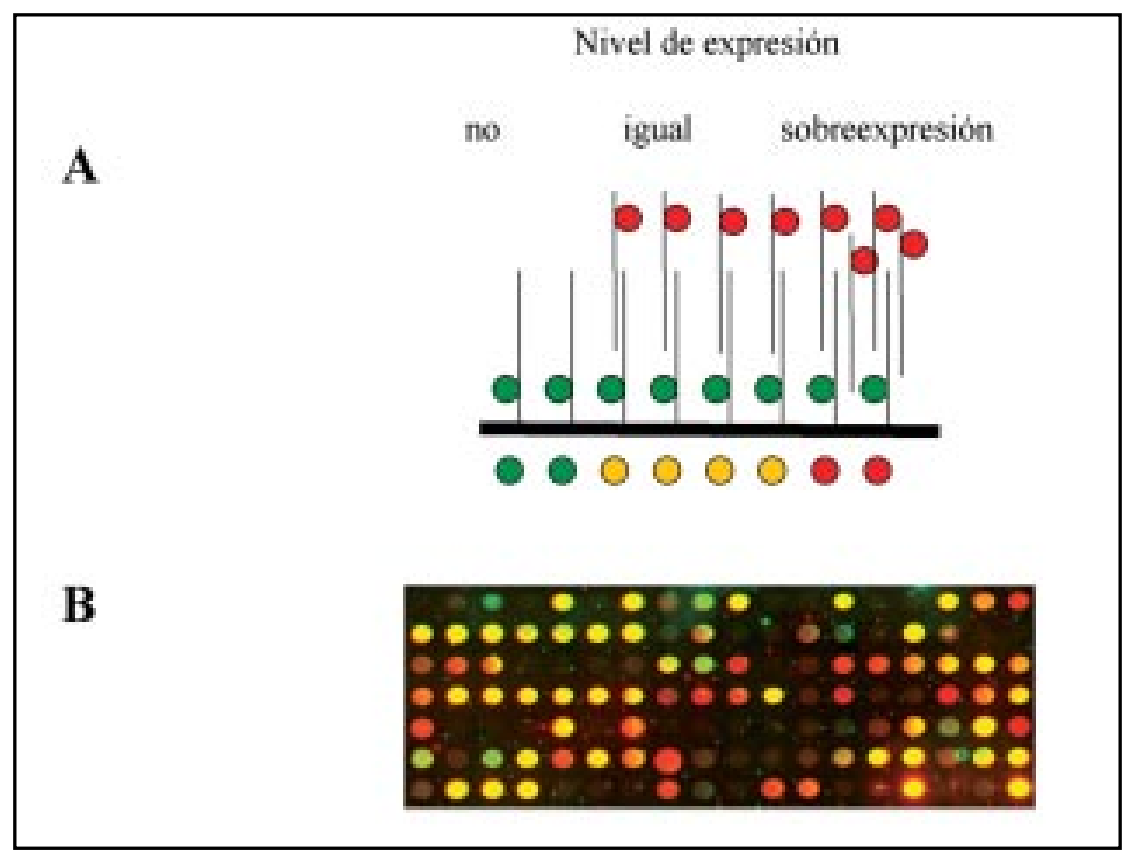

Figura 1. Esquema de cDNA microarray. A) soporte sólido con secuencias impresas del genoma humano (marcadas con FITC) y cDNA de tumores (marcadas con Texasred) y los resultados de la hibridación. En verde, no señal, amarillo, coexpresión y en rojo, sobreexpresión. B) resultado esquemático de un experimento de cDNA microarray, identificando genes con diferentes nivel de expresión (verde, amarillo y rojo).

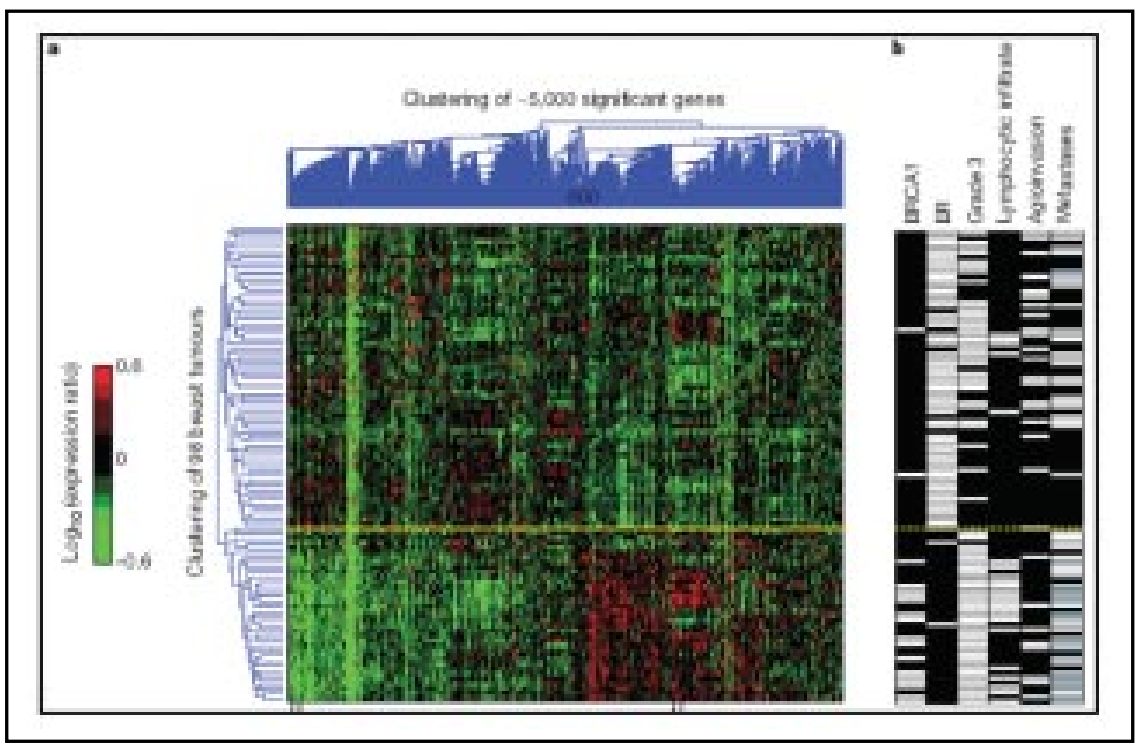

Figura 2. Resultados del perfil de expresión de 5.000 genes en 98 casos de cáncer de mama primario y sus correlaciones clínico-patológicas. A) Análisis de agrupamiento o Heriarchical clustering de los 5.000 genes, muestra dos grandes grupos uno de las cuales se caracteriza por la sobreexpresión de 231 genes (color rojo por debajo de la línea amarilla). B) Correlaciones clínico-patológicas de ambos grupos de tumores demuestra una mayor frecuencia de tumores receptor de estrógenos negativo, alto grado histológico (grado 3), infiltración linfoide y mayor frecuencia de metástasis el grupo con sobreexpresion de los 231 genes. Tomado de van't Veer et al. Nature 2002; 415: 530-535. (con autorización). 
et $\mathrm{al}^{21}$ han propuesto una clasificación molecular del cáncer de mama en la cual distingue i) tumores basales de tipo epitelial (basal epitheliallike group), ii) tumores que sobreexpresan c-erbB2 y iii) tumores de lumen epitelial receptor de estrógenos positivos, la cual tiene significado pronóstico. Por otro lado, van't Veer et $\mathrm{al}^{20}$ estudiaron el perfil de expresión de 25.000 secuencias génicas por cDNA microarray en 78 casos de cáncer esporádicos (34 de las cuales desarrollaron metástasis y 44 que estaban libres de enfermedad a los 5 años de seguimiento) y 20 casos de cáncer de mama hereditario. El análisis de cDNA microarray les permitió identificar al menos 5.000 genes con alteraciones en sus niveles de expresión génica en al menos 3 tumores en forma simultánea. El análisis de agrupamiento jerárquico (Heriarchical Clustering) ${ }^{23}$ de los 5.000 genes generó dos grandes grupos, uno de los cuales se caracterizaba por la sobreexpresión de 231 genes y el otro por la ausencia de sobreexpresion de estos 231 genes (Figura 2). El grupo de sobreexpresión génica se caracterizaba por una mayor frecuencia de tumores receptor de estrógenos negativo, alto grado histológico (grado 3), infiltración linfoide y mayor frecuencia de metástasis. Para acotar el número de genes, responsa- bles de este grupo de mal pronóstico, utilizaron el método Leave-one-out que estima el margen de error al reanalizar grupos de genes en relación todas las muestras ${ }^{24}$, identificando finalmente 70 genes sobreexpresados asociados a mal pronóstico. En un siguiente estudio, este mismo grupo de investigadores analizó la sobreexpresión de estos 70 genes en 295 tumores consecutivos, comparando su valor predictivo de diseminación y metástasis versus parámetros clínico-patológicos tales como edad, tamaño tumoral, compromiso de linfonodos axilares, tipo y grado histológico, y receptores hormonales ${ }^{22}$. Los resultados de este análisis demostraron que el perfil de expresión génica de estos 70 genes era superior a todos los criterios clínico-patológicos tradicionales utilizados en la predicción de diseminación y metástasis

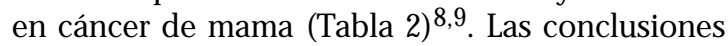
de ambos estudios fue que la información obtenida por cDNA microarray sería el mejor parámetro en la selección de pacientes candidatas a terapias adyuvantes $20,22,25$.

Dada la sugerencia de que el análisis por cDNA microarray mejoraría la selección de pacientes candidatas a terapias adyuvantes, en particular en el grupo de bajo riesgo de recaída a 5 años, se generó el proyecto Translating Molecular

Tabla 2. Análisis multivariado del riesgo de desarrollar metástasis en cáncer de mama

\begin{tabular}{|lcrr|}
\hline Variable & Riesgo relativo & $(95 \%$ CI) & $p$ \\
\hline Perfil de expresion génica & & & \\
de mal pronóstico (vs buen pronóstico) & 4,6 & $(2,3-9,2)$ & $<0,001$ \\
Edad (en interavalos de 10 años) & 0,73 & $(0,50-1,06)$ & 0,10 \\
Estado de linfonodos & 1,13 & $(1,03-1,24)$ & 0,01 \\
Diámetro del tumor (cm) & 1,56 & $(1,22-2,0)$ & $<0,001$ \\
Grado tumoral & & & 0,54 \\
$\quad$ Grado 2 2 (vs grado 1) & 1,35 & $(0,61-3,0)$ & \\
$\quad$ Grado 3 (vs grado 1) & 1,03 & $(0,44-2,4)$ & \\
Invasión vascular & & & \\
$\quad$ 1-3 vasos (vs 0 vasos) & 0,66 & $(0,30-1,44)$ & \\
$\quad$ >3 vasos (vs 0 vasos) & 1,65 & $(0,98-2,8)$ & \\
Receptor de estrógenos & 0,86 & $(0,56-1,31)$ & 0,48 \\
Mastectomía (vs terapia conservadora) & 1,27 & $(0,79-2,0)$ & 0,32 \\
Quimioterapia (vs no quimioterapia) & 0,37 & $(0,20-0,66)$ & $<0,001$ \\
Tratamiento hormonal & & & \\
(vs no tratamiento hormonal) & 0,62 & $(0,29-1,34)$ & 0,23 \\
\hline
\end{tabular}

Tomado de van de Vijver et al. N Engl J Med 2002; 347: 1999-2009. 
Knowledge Into Early Breast Cancer Management del Breast Internacional Group (BIG), un consorcio de 32 grupos de investigación clínica en cáncer de mama en todo el mundo, del cual Chile forma parte a través del Grupo Oncológico Cooperativo Chileno de Investigación (GOCCHI). El proyecto consiste en tres protocolos clínicos de evaluación de perfiles de expresión génica para la predicción de respuesta clínica en cáncer de mama. El primer protocolo se denomina MINDACT (Microarray for Node Negative Disease may Avoid Chemotherapy) y propone comparar parámetros clínico-patológicas versus perfil de expresión génica en forma prospectiva para la decisión de terapias adyuvantes (quimioterapia u hormonoterapia) en pacientes con cáncer de mama y linfonodos axilares negativos. Para ello el MINDACT va a reclutar 8.000 pacientes a través de los 32 grupos cooperativos de investigación afiliados, realizar evaluación clínico-patológica y perfil de expresión génica a todas las pacientes ingresadas $\mathrm{y}$ aleatoriamente someterlas a terapias adyuvantes según criterios de alto o bajo riesgo utilizando ambos métodos (Figura 3). De este modo, pacientes clasificadas como de alto riesgo por paráme- tros clínico-patológicos y perfiles de expresión génica serán candidatas a terapias adyuvantes, (antraciclinas o docetaxel y capecitabina). Las pacientes clasificadas como de bajo riesgo no tendrán indicación de terapias adyuvantes. Por otra parte, las pacientes con discrepancia entre los parámetros clínico-patológicos y perfil de expresión génica, serán randomizadas según uno u otro criterio para recibir o no tratamientos adyuvantes. Por lo tanto, existirá un grupo de pacientes con variables clínico-patológicas de alto riesgo y perfil de expresión génica de bajo riesgo que no recibirán terapia adicional y otro grupo de pacientes con variables clínico-patológicas de bajo riesgo y perfil genético de alto riesgo que si recibirán tratamiento adicional.

El protocolo espera una distribución de 3.300 (55\%) pacientes en la rama de alto riesgo por ambos métodos y 600 (10\%) en la rama de bajo riesgo. En las ramas de discordancia se espera ingresar 2.100 (35\%) pacientes, repartidas en 1.680 (80\%) pacientes con perfil clínico-patológico de alto riesgo y perfil genético de bajo riesgo, y 420 (20\%) pacientes con perfil clínico-patológico de bajo riesgo y perfil genético de alto riesgo. La

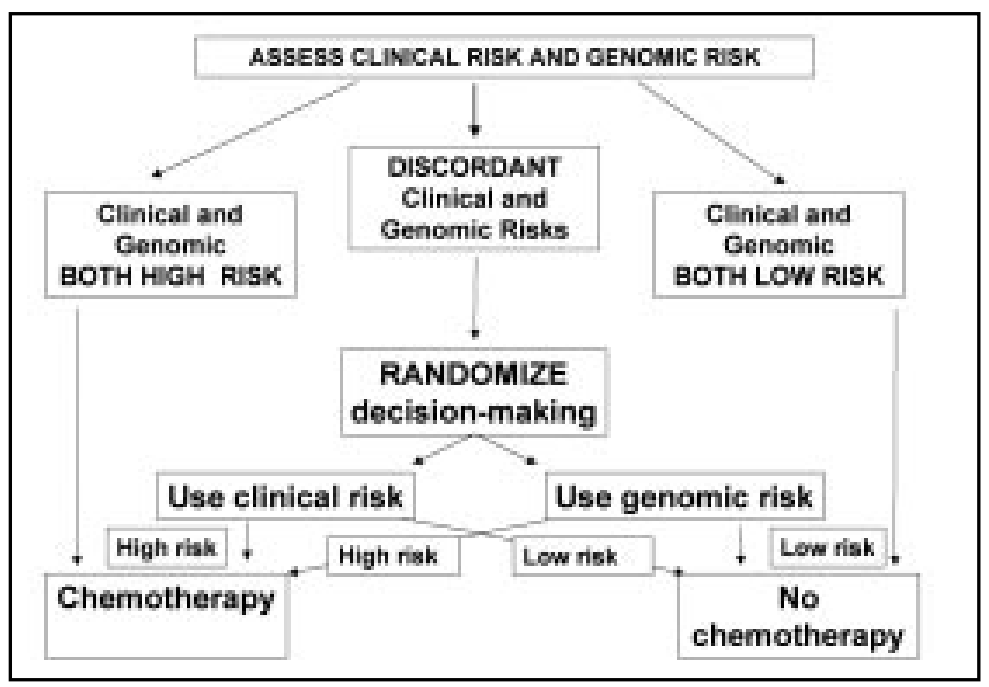

Figura 3. Diseño del protocolo MINDACT para la randomización a quimioterapia según parámetros clínicopatológicos o perfil de expresión génica. Las pacientes con criterios clínicos o de perfil genético de alto riesgo recibirán terapias adyuvantes. Lo contrario ocurrirá para las pacientes con criterios de bajo riesgo por ambos métodos. Las pacientes con criterios clínicos y genéticos discordantes serán randomizadas a recibir o no quimioterapia. 
decisión más conflictiva de este protocolo serán las 1.680 pacientes que no recibirán terapia adyuvante basada en un perfil genético de bajo riesgo, a pesar de poseer parámetros clínicopatológicos de alto riesgo. Este escenario plantea una emergente necesidad de contar con marcadores de recaída de alta sensibilidad y especificidad para el monitoreo de estas pacientes. Para ello, GOCCHI ha propuesto un protocolo adicional de seguimiento para estas pacientes basado en la alteración de expresión de 22 genes (ALDH4, AP2B1, BBC3, CCNE2, CEGP1, CFFM4, DC13, DCK 1, EXT1, FGF18, FLJ11190, FLT, GMPS, GSTM3, HEC, KIAA0175, MCM6, OXCT, PK428, TGFB3, AKAP2, ECT2). Estos genes fueron seleccionados a partir de los 70 genes originalmente identificados en los estudios de van't Veer et $\mathrm{al}^{20}$. El propósito de este protocolo adicional es monitorear las pacientes con perfil de bajo riesgo y parámetros clínico-patológicos de alto riesgo randomizadas durante su seguimiento. En forma preliminar, el análisis de 8 de estos 22 genes en un

\section{REFERENCIAS}

1. PARKIN DM. Global cancer statistics in the year 2000. Lancet Oncol 2001; 2: 533-43.

2. Jensen OM, Esteve J, Moler H, Renard H. Cancer in the European Community and its member states. Eur J Cancer 1990; 26: 1167-256.

3. Jemal A, Tiwari RC, Murray T, Ghafoor A, Samuels A, Ward E et al. Cancer statistics, 2004. CA Cancer J Clin 2004; 54: 8-29.

4. Prieto M. Mortalidad por Cáncer en Chile. Unidad de Cancer, Ministerio de Salud 2000.

5. Bonadonna G, Valagussa P. Current status of adjuvant chemotherapy for breast cancer. Semin Oncol 1987; 14: 8-22.

6. Consensus Conference Adjuvant chemotherapy for breast cancer. JAMA 1985; 254: 3461-3.

7. Bonadonna G. Evolving concepts in the systemic adjuvant treatment of breast cancer. Cancer Res 1992; 52: 2127-37.

8. Goldhirsch A, Glick JH, Gelber RD, Senn HJ. Meeting highlights: International Consensus $\mathrm{Pa}-$ grupo piloto de 10 casos ( 5 de ellos sin metástasis ganglionares y 5 con metástasis ganglionares) demostró que al menos 3 genes (BBC3, HEC y DC13) caen de manera significativa en las pacientes con metástasis ganglionares (observaciones no publicadas). Este hallazgo permitiría plantear la posibilidad de seguimiento molecular en pacientes no randomizadas a tratamientos adyuvantes.

La incorporación del perfil de expresión génica en la clasificación de riesgo en pacientes con cáncer de mama y linfonodos axilares negativos constituirá una revolución en el manejo clínico en la oncología en general y en cáncer de mama en particular. El protocolo MINDACT es probablemente el primer esfuerzo prospectivo de evaluar la utilidad de marcadores genéticos en decisiones clínicas. La oportunidad de participar en este protocolo a pacientes chilenas a través de GOCCHI, nos permitirá estar en la primera línea de la investigación de nuevos métodos para la toma de decisiones terapéuticas que potencialmente pueden cambiar la oncología actual. nel on the Treatment of Primary Breast Cancer. J Natl Cancer Inst 1998; 90: 1601-8.

9. Eifel P, Axelson Ja, Costa J, Crowley J, Curran WJ JR, Deshier A ET AL. National Institutes of Health Consensus Development Conference Statement: adjuvant therapy for breast cancer. J Natl Cancer Inst 2001; 93: 979-89.

10. Jatoi I, Hilsenbeck SG, Clark GM, Osborne CK. Significance of axillary lymph node metastasis in primary breast cancer. J Clin Oncol 1999; 17: 2334-40.

11. Colozza M, Cardoso F, Sotiriou C, Larsimont D, PICCART MJ. Bringing molecular prognosis and prediction to the clinic. Clin Breast Cancer 2005; 6: 61-76.

12. SUBRAMANIAM DS, ISAACS C. Utilizing prognostic and predictive factors in breast cancer. Curr Treat Options Oncol 2005; 6: 147-59.

13. Ross JS, Linette GP, Stec J, Ciark E, Ayers M, LeSCHLY N ET AL. Breast cancer biomarkers and molecular medicine. Expert Rev Mol Diagn 2003; 3: 573-85. 
14. Mocelun S, Provenzano M, Rossi CR, Pilati P, Nitti D, LISE M. DNA array-based gene profiling: from surgical specimen to the molecular portrait of cancer. Ann Surg 2005; 241: 16-26.

15. Bucca G, Carruba G, Saetta A, Muti P, Castagnetta L, Sмттн CP. Gene expression profiling of human cancers. Ann NY Acad Sci 2004; 1028: 28-37.

16. KIm WH, RuBin MA, DunN RL. High-density tissue microarray. Am J Surg Pathol 2002; 26: 1236-8.

17. Staudt LM. Gene expression profiling of lymphoid malignancies. Annu Rev Med 2002; 53: 30318.

18. Petty RD, Nicolson MC, Kerr KM, Cowe-Duguid E, Murray GI. Gene Expression Profiling in NonSmall Cell Lung Cancer: From Molecular Mechanisms to Clinical Application. Clin Cancer Res 2004; 10: 3237-48.

19. Perou CM, Sorde T, Eisen MB, van de Ruj M, JefFrey SS, ReES CA et aL. Molecular portraits of human breast tumours. Nature 2000; 406: 747-52.

20. van't Veer LJ, Dai H, van de ViJver MJ, He YD, Hart AA, MAO M ET AL. Gene expression profiling predicts clinical outcome of breast cancer. Nature 2002; 415: 530-6.

21. Sorlie T, Perou CM, Tibshirani R, Aas T, Geisler S, Johnsen H ET AL. Gene expression patterns of breast carcinomas distinguish tumor subclasses with clinical implications. Proc Natl Acad Sci USA 2001; 98: 10869-74.

22. van de ViJver MJ, He YD, van't VeER LJ, Dai H, HaRT AA, VoskuIL DW ET AL. A gene-expression signature as a predictor of survival in breast cancer. $\mathrm{N}$ Engl J Med 2002; 347: 1999-2009.

23. Eisen MB, Speluman PT, Brown PO, Botstein D. Cluster analysis and display of genome-wide expression patterns. Proc Natl Acad Sci USA 1998; 95: 14863-8.

24. ReDelMEIER DA, TibShIRANI RJ. Interpretation and bias in case-crossover studies. J Clin Epidemiol 1997; 50: 1281-7.

25. Tuma RS. A big trial for a new technology: TransBIG Project takes microarrays into clinical trials. J Natl Cancer Inst 2004; 96: 648-9. 